\title{
Clinical optimal dose of solifenacin succinate for nursing patients after transurethral resection of the prostate during the perioperative period
}

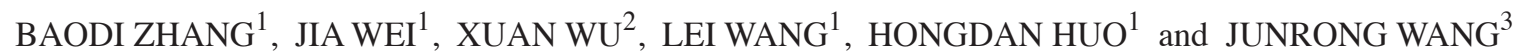 \\ ${ }^{1}$ Department of Surgery, ${ }^{2}$ Dialysis Room and ${ }^{3}$ Operating Room, First Branch of Hongqi Hospital \\ of Mudanjiang Medical University, Mudanjiang, Heilongjiang 157011, P.R. China
}

Received February 16, 2016; Accepted April 21, 2017

DOI: $10.3892 /$ etm.2017.5567

\begin{abstract}
Transurethral plasma kinetic resection is an efficient and safe surgery for the treatment of benign prostatic hyperplasia. Solifenacin succinate (SOL) is safe and clinically efficient for patients who endure transurethral resection of the prostate (TURP) during the perioperative period. The objective of this study was to evaluate the clinical optimal dose of SOL for nursing patients after TURP during the perioperative period. Patients were recruited and randomized into three groups: SOL (3 mg), SOL (6 mg), and SOL (10 mg). All patients received medical care for 3 weeks after TURP. Levels of inflammatory cytokines, including IL-6, epithelial neutrophil activation peptide-7 (ENA-7), tumor necrosis factor (TNF)- $\alpha$, interleukin (IL)-2, IL-17 and IL-8, were investigated in the patients of all three groups. The efficacy of SOL was analyzed via the following scores: International Prostate Symptom Score (IPSS), Overactive Bladder Symptom Score (OBSS), short-form voiding (SFV) and storage score (TS) of International Continence Society (ICS). Outcomes showed that IL-2 and ENA-7 plasma concentration levels were upregulated, whereas TNF- $\alpha$, IL-6, IL-17 and IL-8 were downregulated, in all three groups. The findings showed that patients that received SOL $(6 \mathrm{mg})$ exhibited significant improvements compared to the other patient groups from baseline to the end of treatment, as determined by IPSS, OBSS, ICS, SFV, TS (P<0.01). In conclusion, these results indicate that SOL $(6 \mathrm{mg})$ is the optimal dose for patients who undergo TURP during the perioperative period. Notably, treatment with SOL (6 mg) exhibited significant additional benefits in terms of lower urinary tract symptoms during the early recovery period after TURP, suggesting SOL is clinically significant for nursing patients who suffer have undergone TURP during the perioperative period.
\end{abstract}

Correspondence to: Professor Junrong Wang, Operating Room, First Branch of Hongqi Hospital of Mudanjiang Medical University, 2 Taiping Road, Mudanjiang, Heilongjiang 157011, P.R. China

E-mail: wangjunrongmdj@sina.com

Key words: solifenacin succinate, transurethral resection of the prostate, inflammation, benign prostate hyperplasia, clinical nursing

\section{Introduction}

Prostatic hyperplasia is a common disease in elderly males that is increasing as the global population ages (1). Clinical manifestations of prostatic hyperplasia are frequent micturition, urgency, incontinence, urinary tract infection, impairments of renal function and inflammation of the prostate gland (2). Treatments of benign prostatic hyperplasia are changing due to a greater understanding of this disease and the development of the functional concept of lower urinary tract symptoms (3). Currently, transurethral resection of the prostate (TURP) is one of the conventional surgical methods for benign prostate hyperplasia that reduces the postoperative recovery time and decreases intraoperative bleeding as well as avoids the damage to prostate (4).

Solifenacin succinate (SOL) is safe and clinically efficient for patients who undergo TURP during the perioperative period (5). Research has found that SOL is efficacious for women with overactive bladder syndrome based on the results of a randomized controlled crossover study (6). Huo et al (7) suggested that a combination of SOL and naftopidil effectively relieves the symptoms of female overactive bladder and improves the patient's quality of life in a randomized controlled crossover study. Notably, SOL facilitated early recovery from incontinence and prevented worsening of quality-of-life during the recovery of voiding dysfunction after radical prostatectomy in men with clinically localized prostate cancer in a prospective, randomized, controlled study (8).

The majority of previous reports have focused on the effects of SOL on TURP and results showed that SOL improved quality-of-life score, overactive bladder symptom score (OBSS), and short-form voiding (SFV) and storage score (TS); therefore, there have been few reports on the optimal dose of SOL for nursing patients who have undergone TURP during the perioperative period. In this study, we investigated the efficacy of SOL in patients who underwent TURP during the perioperative period.

\section{Patients and methods}

Ethics statement. The clinical design of the present study was approved by the ethics committee of the First Branch of Hongqi Hospital of Mudanjiang Medical University (approval 
no., 20120514RD; Mudanjiang, China). All patients with benign prostate hyperplasia provided written informed consent prior to treatment.

Study design and patients. This randomized study was conducted in 246 men with benign prostate hyperplasia who underwent TURP. Patients were orally treated with SOL (Sigma-Aldrich; Merck KGaA, Darmstadt, Germany; 3, 6 or $10 \mathrm{mg}$ ) 1 week prior to TURP and 2 weeks after TURP. Inclusion criteria at baseline and the endpoints of treatment were determined by the following clinical scores: International Prostate Symptom Score (IPSS), $\geq 8$; voided volume, $\geq 125 \mathrm{ml}$; maximum urinary flow rate $\left(Q_{\max }\right)$, $\leq 15 \mathrm{ml} / \mathrm{s}$; and prostate volume, $\geq 30 \mathrm{ml}$ and $<100 \mathrm{ml}$ (9).

Grouping and treatment. A total of 246 male patients with benign prostate hyperplasia were screened using previously reported guidelines (10) and randomly divided into three groups as follows: $3 \mathrm{mg}$ SOL $(\mathrm{n}=80), 6 \mathrm{mg} \mathrm{SOL}(\mathrm{n}=88)$ and $10 \mathrm{mg}$ SOL $(\mathrm{n}=76)$. The ages of the patients ranged from 46.2 to 66.4 years. Patients in all three groups orally received treatment once daily for 3 weeks.

ELISA. Serum levels of IL-8 (KHC0081), IL-1 (MBS700340), IL-17 (88-7876-88), TNF- $\alpha$ (KHC3014), IL-6 (KHC0062), and ENA-7 (KHC1164; all Thermo Fisher Scientific, Inc., Waltham, MA, USA) were detected in patients with benign prostate hyperplasia prior to and following treatment with SOL using respective ELISA kits (Thermo Fisher Scientific, Inc.) according to the manufacturer's instructions. Finally, the serum concentration levels of the inflammatory cytokines were measured by using a micro-plate reader at $450 \mathrm{~nm}$.

Efficacy assessments. Baseline and endpoint characteristics of patients were analyzed via TS, total IPSS, IPSS storage, voiding scores, quality of life (QoL) score, total OABSS questionnaire, and SFV of ICS (11). Mean measurements were calculated at baseline and at the endpoint of treatment after TURP for patients with benign prostate hyperplasia. Micturition diary variables were determined by volume voided (Vvoid) per micturition and safety parameters were analyzed by adverse events, post-void residual (PVR). and $Q_{\max }$.

Statistical analysis. Statistical analyses of parameters were expressed as the mean \pm standard deviation of triplicate dependent experiments and were analyzed using one-way analysis of variance followed by Tukey's post hoc test for all patients who completed this clinical study. Statistical calculations were analyzed using SPSS 19.0 (IBM Corp., Armonk, NY, USA) and GraphPad Prism version 5.0 (GraphPad Software, Inc., La Jolla, CA, USA). $\mathrm{P}<0.05$ was considered to indicate a statistically significant difference.

\section{Results}

Characteristics of patients. A total of 246 men with benign prostate hyperplasia were screened and randomly divided into three groups (3 mg SOL, $\mathrm{n}=80 ; 6 \mathrm{mg} \mathrm{SOL}, \mathrm{n}=88 ; 10 \mathrm{mg} \mathrm{SOL}$, $\mathrm{n}=76$ ). Characteristics of patients were summarized in Table I. There were no significant differences in baseline characteristics among the three groups. The age of patients with benign
Table I. Characteristic of patients and statistical data at baseline.

\begin{tabular}{lccc}
\hline Parameters & $\begin{array}{c}\text { SOL } \\
(3 \mathrm{mg})\end{array}$ & $\begin{array}{c}\text { SOL } \\
(6 \mathrm{mg})\end{array}$ & $\begin{array}{c}\text { SOL } \\
(10 \mathrm{mg})\end{array}$ \\
\hline Number & 80 & 88 & 76 \\
Age, years & $46.2-57.4$ & $50.4-59.5$ & $28.8-66.4$ \\
IPSS total score & $-7.66 \pm 1.82$ & $-7.48 \pm 2.02$ & $-7.62 \pm 1.86$ \\
OABSS total score & $0.54 \pm 0.86$ & $0.44 \pm 0.94$ & $0.53 \pm 0.90$ \\
PVR & $-1.43 \pm 6.12$ & $-1.58 \pm 5.84$ & $-1.48 \pm 5.76$ \\
$Q_{\max }$ & $6.98 \pm 1.48$ & $6.84 \pm 1.76$ & $6.95 \pm 1.88$ \\
\hline
\end{tabular}

SOL, solifenacin succinate; IPSS, International Prostate Symptom Score; OABSS, Overactive Bladder Symptom Score; PVR, post-void residual; $Q_{\max }$, maximum urinary flow rate.

Table II. Treatment-related adverse events of SOL for patients during perioperative period.

\begin{tabular}{lrcc}
\hline & $\begin{array}{r}\text { SOL } \\
(3 \mathrm{mg} ; \\
\mathrm{n}=60)\end{array}$ & $\begin{array}{c}\text { SOL } \\
(6 \mathrm{mg} ; \\
\mathrm{n}=68)\end{array}$ & $\begin{array}{c}\text { SOL } \\
(10 \mathrm{mg} ; \\
\mathrm{n}=54)\end{array}$ \\
\hline Parameters & 8 & 10 & 16 \\
Constipation & 11 & 12 & 15 \\
Urinary retention & 0 & 0 & 2 \\
Decreased appetite & 5 & 7 & 9 \\
Hypertriglyceridemia & 6 & 6 & 8 \\
Fatigue & 4 & 5 & 7 \\
\hline
\end{tabular}

Data are presented as the number of adverse events. SOL, solifenacin succinate.

prostate hyperplasia ranged from 46.2 to 66.4 years. In all groups, the range of the total IPSS was 14.44 to 16.64 and the range of the SFV was 146.36 to $174.86 \mathrm{ml}$ at baseline.

Efficacy of SOL on inflammatory cytokine levels. The efficacy of different doses of SOL on inflammatory cytokine levels in serum was investigated in patients prior to and following treatment with SOL. The findings demonstrated that serum levels of TNF- $\alpha$, IL- 6 , IL-17 and IL- 8 were significantly downregulated in the 6 and $10 \mathrm{mg}$ SOL groups compared with the $3 \mathrm{mg}$ SOL group (Fig. 1A-D); whereas serum levels of IL-2 and ENA-7 were upregulated in the 6 and $10 \mathrm{mg}$ SOL groups (Fig. 1E and F). These dose-dependent differences were significant. These results suggest that higher doses of SOL (6 and $10 \mathrm{mg}$ ) are more efficient compared with the lower dose of SOL (3 mg) for decreasing levels of adverse inflammatory cytokines and increasing levels of beneficial inflammatory cytokines in patients who have undergone TURP.

Efficacy of SOL on patients with benign prostate hyperplasia. The findings demonstrated that, compared with the $3 \mathrm{mg}$ of SOL group, the 6 and $10 \mathrm{mg}$ SOL groups exhibited 
A

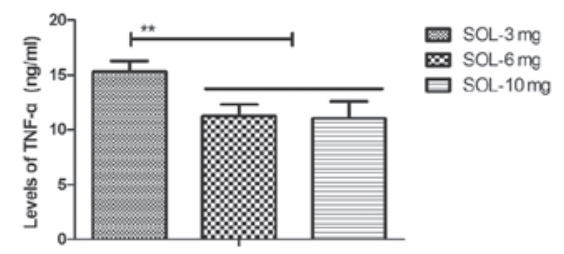

D

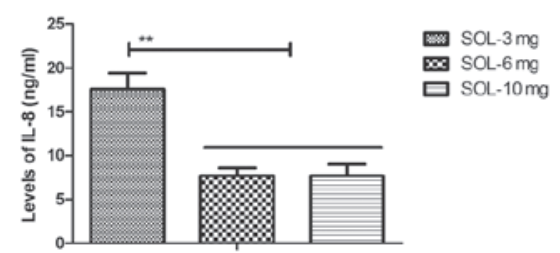

B

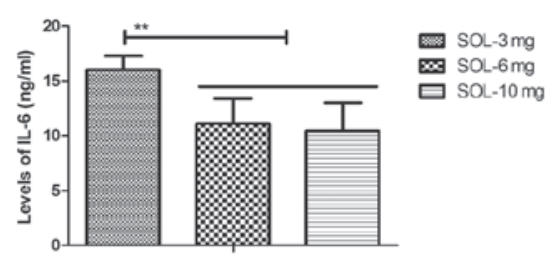

$\mathbf{E}$

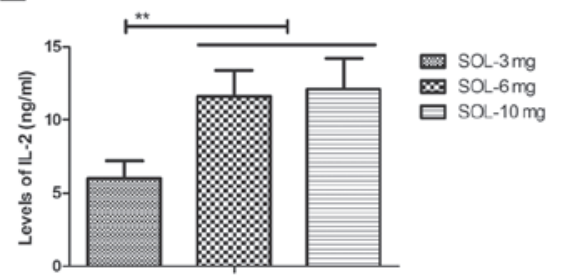

C

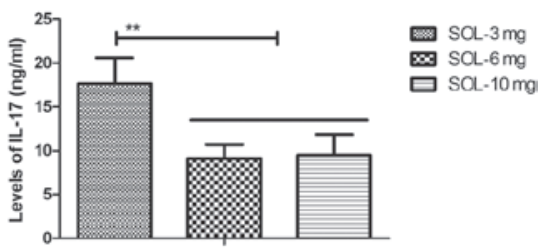

$\mathbf{F}$

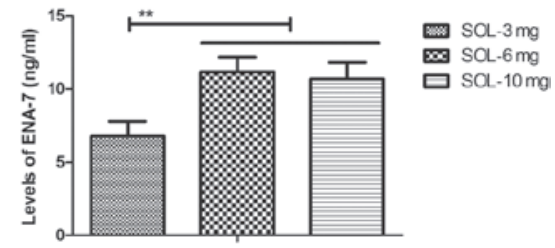

Figure 1. SOL treatment decreases inflammatory cytokines in patients with benign prostatic hyperplasia who undergone TURP. SOL treatment decreases serum levels of (A) TNF- $\alpha$, (B) IL-6, (C) IL-17 and (D) IL-8 in patients with benign prostatic hyperplasia who have undergone TURP. (E and F) SOL treatment decreases serum levels of (E) IL-2 and (F) ENA-7 in patients with benign prostatic hyperplasia who have undergone TURP. *P $<0.01$ as indicated. SOL, solifenacin succinate; TNF, tumor necrosis factor; IL, interleukin; TURP, transurethral resection of the prostate; ENA-7, epithelial neutrophil activation peptide-7.

A

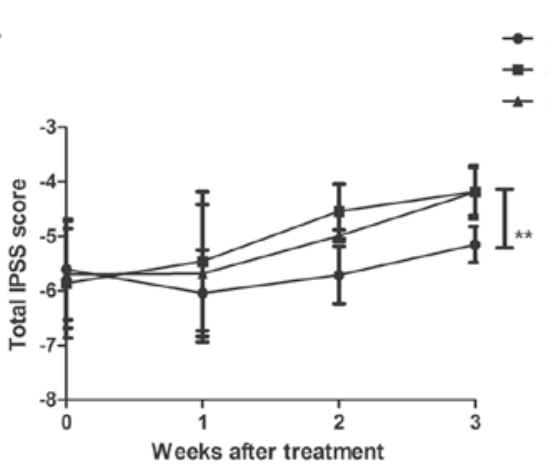

C

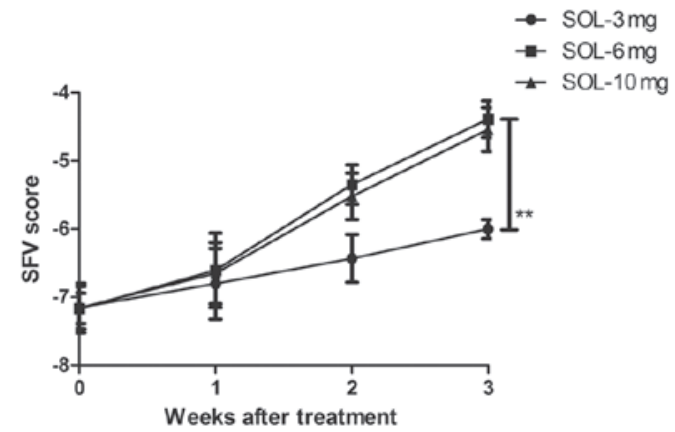

B

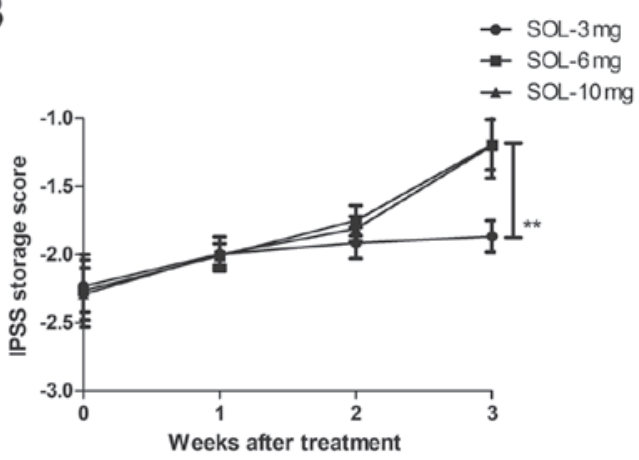

D
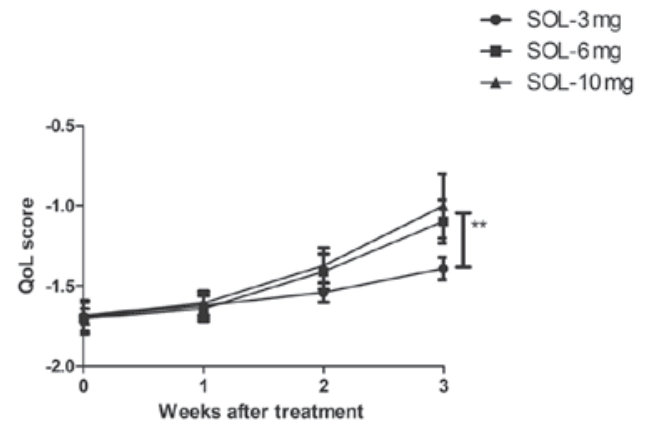

Figure 2. Efficacy of SOL on patients with benign prostate hyperplasia. (A) SOL treatment significantly improves total IPSS score of patients with benign prostatic hyperplasia who have undergone TURP. SOL treatment improves (B) IPSS storage, (C) SFV and (D) IPSS quality of life scores of patients with benign prostatic hyperplasia who have undergone TURP. ${ }^{* *} \mathrm{P}<0.01$. SOL, solifenacin succinate; TURP, transurethral resection of the prostate; IPSS, International Prostate Symptom Score; SFV, short-form voiding.

significantly decreased total IPSS scores after two weeks of treatment (Fig. 2A). As shown in Fig. 2B-D, IPSS storage, SFV and IPSS QoL scores were increased after 3 weeks of medical care compared with the baseline (week 0). Notably, the 6 and $10 \mathrm{mg}$ of SOL groups exhibited significant differences when compared with the $3 \mathrm{mg}$ SOL group. In addition, OBASS total score was improved, evidenced by the mean decrease from baseline (week 0) to the end of treatment (week 3) in three groups (Fig. 3A). ICS voiding score and ICS storage score were increased by SOL treatment in the three groups after 3 weeks of treatment; however, ICS voiding score and ICS storage score were not significantly different between the $6 \mathrm{mg}$ and $10 \mathrm{mg}$ groups after 3 weeks of treatment (Fig. 3B and C). Furthermore, compared with baseline (week 0), improvements 

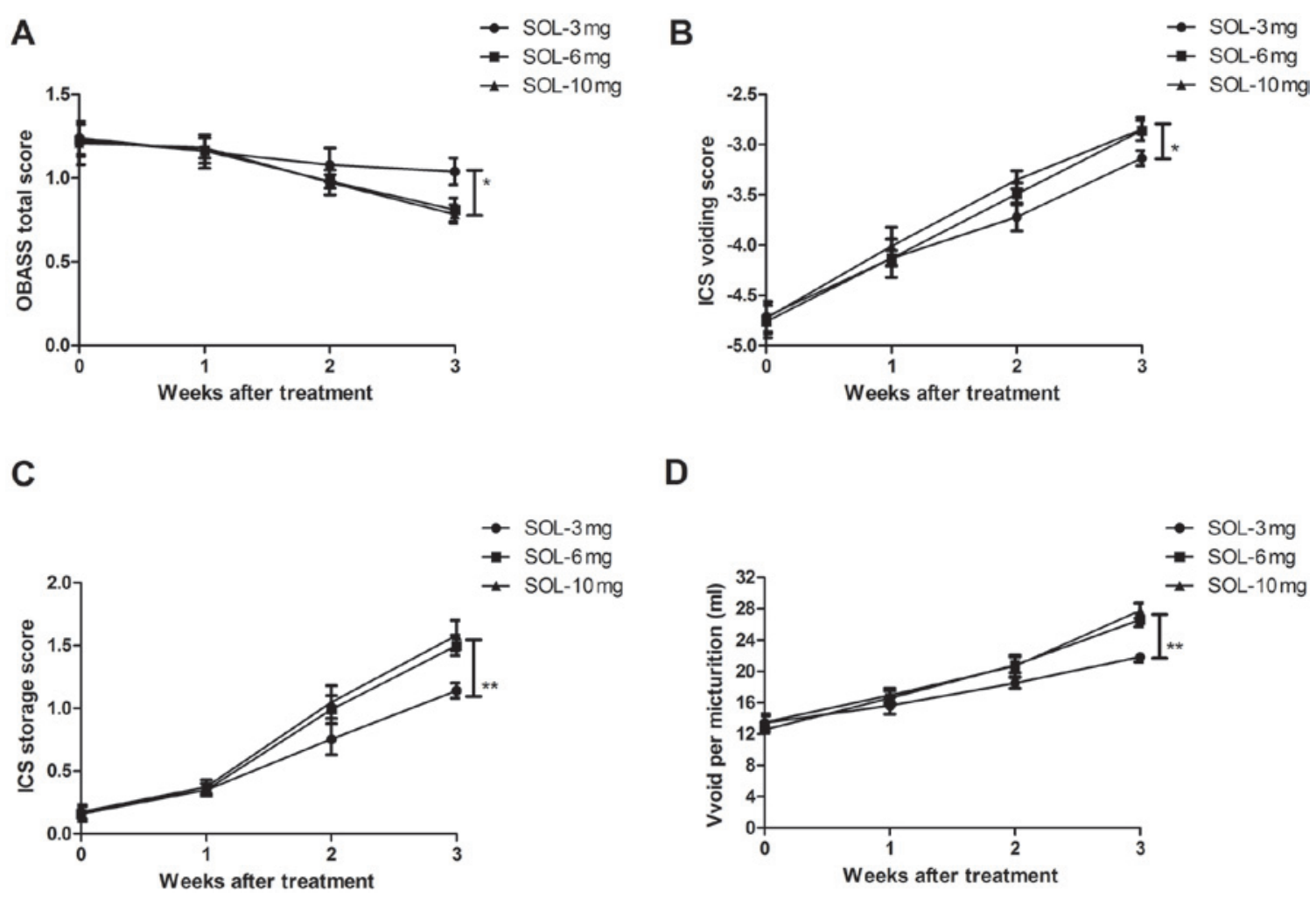

D

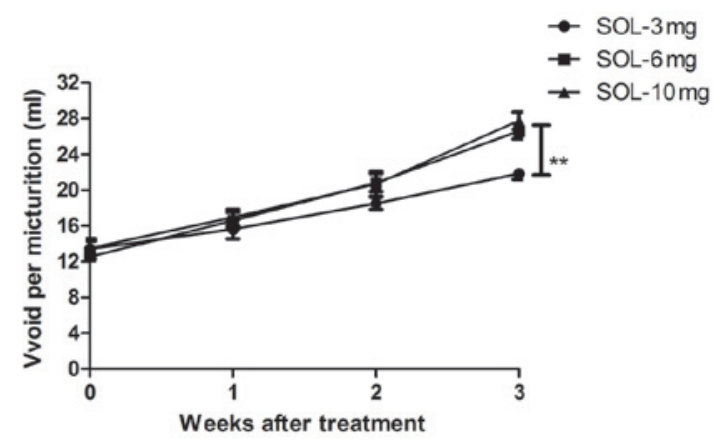

Figure 3. SOL treatment improves ICS score for patients with benign prostate hyperplasia. (A) SOL treatment significantly improves OBASS total score of patients with benign prostatic hyperplasia who have undergone TURP. SOL treatment increases (B) ICS voiding score and (C) ICS storage score of patients with benign prostatic hyperplasia who have undergone TURP. (D) SOL treatment increases Vvoid per micturition for patients with benign prostate hyperplasia during perioperative period. ${ }^{*} \mathrm{P}<0.05,{ }^{* *} \mathrm{P}<0.01$. SOL, solifenacin succinate.

\section{A}

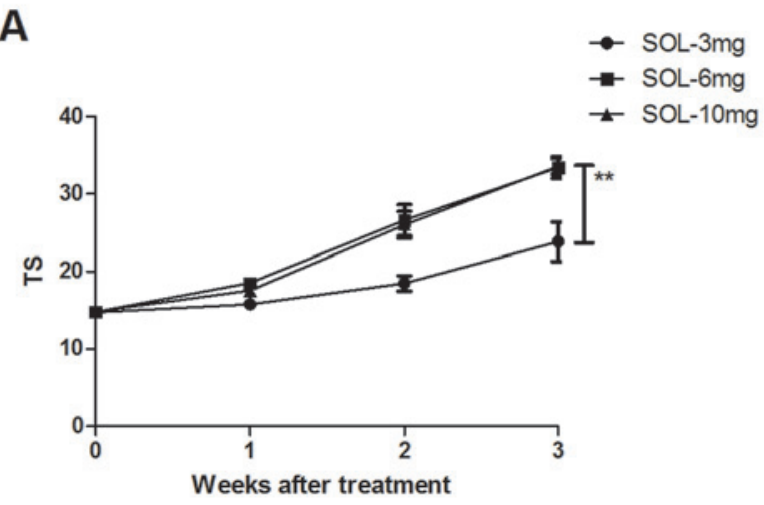

B

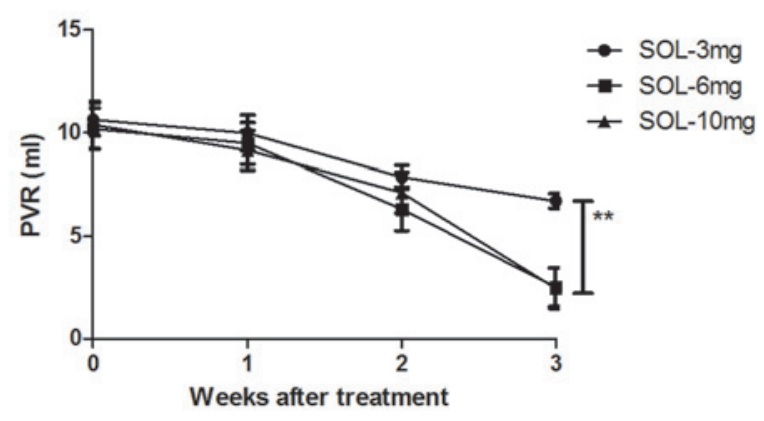

Figure 4. Safety of SOL for patients with benign prostate hyperplasia. Mean changes of patients with benign prostate hyperplasia during perioperative period from baseline determined by (A) TS and (B) PVR during 3-week treatment. ${ }^{* *} \mathrm{P}<0.01$. SOL, solifenacin succinate; Vvoid, volume voided; PVR, post-void residual; TS, storage score.

in Vvoid per micturition were noted after 3 weeks of treatment in the three groups, and the 6 and $10 \mathrm{mg}$ SOL groups exhibited a significant difference when compared with the $3 \mathrm{mg} \mathrm{SOL}$ group (Fig. 3D). These outcomes suggest that SOL treatment is efficient for patients with benign prostate hyperplasia during perioperative period.

Safety of SOL for patients with benign prostate hyperplasia. Finally, 68 patients that received $3 \mathrm{mg}$ SOL, 60 patients administered $6 \mathrm{mg}$ SOL and 54 patients given $10 \mathrm{mg}$ SOL completed this study. Outcomes showed that mean changes from baseline in Vvoid per micturition were increased in three groups. Notably, patients in the two groups that received 6 and $10 \mathrm{mg}$ SOL, respectively, exhibited a significant difference when compared with patients in the $3 \mathrm{mg}$ SOL group, as determined by mean changes in TS from baseline to the end of treatment (Fig. 4A). By the end of treatment (week 3), mean PVR values were significantly improved following treatment with 6 and $10 \mathrm{mg}$ SOL, respectively, as compared with $3 \mathrm{mg}$ SOL (Fig. 4B). All adverse events are summarized in Table II. The most common adverse events were dry mouth and constipation after SOL treatment. These results suggest that SOL is a safe agent for the treatment of patients with benign prostate hyperplasia. 


\section{Discussion}

Prostate hyperplasia is a disease that often occurs in elderly men and is caused by pathophysiological changes after urinary tract obstruction (12). Previous reports have shown that SOL is a safe and efficient treatment for patients with benign prostate hyperplasia after transurethral resection of the prostate $(8,13)$. Reports also suggest that TURP is low risk, highly safe and associated with less complications when treating benign prostate hyperplasia $(14,15)$. In this study, we investigated the efficacy of different dosages of SOL for patients with benign prostate hyperplasia during the perioperative period. We first analyzed the efficacy of SOL on inflammatory cytokines in patients with benign prostate hyperplasia after TURP. The findings showed that SOL significantly decreased serum levels of TNF- $\alpha$, IL-6, IL-17 and IL-8, and increased serum levels of IL-2 and ENA-7, in the three groups. Outcomes also suggest that SOL treatment exhibited additional benefits in terms of total IPSS, IPSS voiding and storage, OABSS total score and Vvoid per micturition. Notably, treatment with $6 \mathrm{mg}$ SOL was demonstrated to be a reliable dosage that had relatively fewer potential side effects than $10 \mathrm{mg}$ of SOL, as determined by PVR and the most common adverse events.

Various drugs, such as antimicrobials and $\alpha$-blockers are available for patients after TURP due to benign prostate hyperplasia $(16,17)$. SOL is a novel muscarinic receptor antagonist and previous findings have suggested that SOL is able to improve detrusor overactivity without causing urinary retention (18). The pharmacological and clinical profile of SOL has indicated that SOL may be a promising drug in the treatment of patients with overactive bladder syndrome, which could be regarded as a novel therapeutic agent for patients with an overactive bladder $(19,20)$. Notably, Shin et al (13) have presented the efficacy and safety of tamsulosin hydrochloride $(0.2 \mathrm{mg})$ and combination therapy with tamsulosin hydrochloride $(0.2 \mathrm{mg})$ plus SOL $(5 \mathrm{mg})$ for patients with benign prostate hyperplasia after TURP in a prospective and randomized controlled trial. In the present study, we investigated the efficacy of different doses of SOL, as demonstrated by the levels of inflammatory cytokines, and researched the clinical importance of SOL for nursing patients after TURP during the perioperative period. Findings suggest that SOL treatment not only decreases IPSS total score, but also improves OABSS total score for patients after TURP. Furthermore, SOL treatment significantly decreased prostate inflammation in patients with benign prostatic hyperplasia, which may be further contributed to recovery during perioperative period after TURP.

A previous review systematically and comprehensively analyzed medical and surgical treatment modalities for lower urinary tract symptoms in a male patient with benign prostatic hyperplasia after surgery (21). Bishr et al (22) also investigated the medical management of benign prostatic hyperplasia in a population-based study. Assessing inflammation and the most common adverse events is essential for nursing patients with benign prostate hyperplasia during the perioperative period $(23,24)$. This study focused on the clinical significance of different dosages of SOL for patients with benign prostate hyperplasia during perioperative period. The outcomes indicated that SOL (6 mg) treatment improved inflammation and is associated with additional benefits (reduced urinary tract symptoms) for patients during the early recovery period after TURP. Safety investigations showed that SOL $(6 \mathrm{mg})$ treatment was associated with minimal side effects. Urinary retention was not observed in the $6 \mathrm{mg}$ group; however, 2 patients in the $10 \mathrm{mg}$ SOL group exhibited urinary retention.

In conclusion, this clinical investigation was the first study to demonstrate that SOL (6 mg) is able to improve inflammation by decreasing the levels of inflammatory cytokines (TNF- $\alpha$, IL-6, IL-17 and IL-8) and increasing serum levels of IL-2 and ENA-7. In addition, SOL treatment improved the urinary tract symptoms during the early recovery period after TURP. To the best of our knowledge, the present study is the first to report a prospective, randomized trial that analyzed the safety and efficacy of SOL for inflammation during the perioperative period. However, further studies should be performed in a larger cohort of patients with benign prostatic hyperplasia after TURP.

\section{References}

1. Shore N: A review of the prostatic urethral lift for lower urinary tract symptoms: Symptom relief, flow improvement and preservation of sexual function in men with benign prostatic hyperplasia. Curr Bladder Dysfunct Rep 10: 186-192, 2015.

2. Zhang W, Yu J, Liu Z and Peng W: Acupuncture for benign prostatic hyperplasia: A systematic review protocol. BMJ Open 5: e007009, 2015.

3. Carrero-López VM, Cózar-Olmo JM and Miñana-López B: Benign prostatic hyperplasia and lower urinary tract symptoms. A review of current evidence. Actas Urol Esp 40: 288-294, 2016.

4. Carnevale FC, Iscaife A, Yoshinaga EM, Moreira AM, Antunes AA and Srougi M: Transurethral resection of the prostate (TURP) versus original and PErFecTED prostate artery embolization (PAE) due to benign prostatic hyperplasia (BPH): Preliminary results of a single center, prospective, urodynamic-controlled analysis. Cardiovasc Intervent Radiol 39: 44-52, 2016.

5. Chu F, Smith N and Uchida T: Efficacy and safety of solifenacin succinate $10 \mathrm{mg}$ once Daily: A multicenter, phase III, randomized, double-blind, placebo-controlled, parallel-group trial in patients with overactive bladder. Curr Ther Res Clin Exp 70: 405-420, 2009.

6. Vecchioli-Scaldazza C, Morosetti C, Berouz A, Giannubilo W and Ferrara V: Solifenacin succinate versus percutaneous tibial nerve stimulation in women with overactive bladder syndrome: Results of a randomized controlled crossover study. Gynecol Obstet Invest 75: 230-234, 2013.

7. Huo LZ, Jing HG, Wang TC, Yuan SX, Luan XH, Guo KC and Shi BK: A combination of solifenacin succinate and naftopidil in the treatment of female overactive bladder. Zhonghua Yi Xue Za Zhi 93: 3526-3528, 2013 (In Chinese).

8. Shim M, Kim J, Park S, Choi SK, Lee SM, Huh KO, Song C, Choo MS and Ahn H: The therapeutic effect of solifenacin succinate on the recovery from voiding dysfunction after radical prostatectomy in men with clinically localized prostate cancer: A prospective, randomized, controlled study. Urology 85: 1123-1129, 2015.

9. Wessels SG and Heyns CF: Prospective evaluation of a new visual prostate symptom score, the international prostate symptom score and uroflowmetry in men with urethral stricture disease. Urology 83: 220-224, 2014.

10. Foo KT: Diagnosis and treatment of benign prostate hyperplasia in Asia. Transl Androl Urol 4: 478-483, 2015.

11. Bunyavejchevin S: Reliability of thai-version overactive bladder symptom scores (OABSS) questionnaire and the correlations of OABSS with voiding diary, international prostate symptom score (IPSS) and patient perception of bladder condition (PPBC) questionnaires. J Med Assoc Thai 98: 1064-1074, 2015.

12. Yoo C, Oh CY, Kim SJ, Kim SI, Kim YS, Park JY, Seong do H, Song YS, Yang WJ, Chung HC, et al: Preoperative clinical factors for diagnosis of incidental prostate cancer in the era of tissue-ablative surgery for benign prostatic hyperplasia: A korean multi-center review. Korean J Urol 53: 391-395, 2012. 
13. Shin YS, Zhang LT, You JH, Choi IS, Zhao C and Park JK Efficacy and safety of tamsulosin hydrochloride $0.2 \mathrm{mg}$ and combination of tamsulosin hydrochloride $0.2 \mathrm{mg}$ plus solifenacin succinate $5 \mathrm{mg}$ after transurethral resection of the prostate: A prospective, randomized controlled trial. Clin Interv Aging 11: 1301-1307, 2016

14. Świniarski PP, Stępień S, Dudzic W, Kęsy S, Blewniewski M and Różański W: Thulium laser enucleation of the prostate (TmLEP) vs. transurethral resection of the prostate (TURP): Evaluation of early results. Cent European J Urol 65: 130-134, 2012.

15. Donovan MJ, Khan FM, Bayer-Zubek V, Powell D, Costa J and Cordon-Cardo C: A systems-based modelling approach using transurethral resection of the prostate (TURP) specimens yielded incremental prognostic significance to Gleason when predicting long-term outcome in men with localized prostate cancer. BJU Int 109: 207-213, 2012.

16. Hennenfent BR, Lazarte AR and Feliciano AE Jr: Repetitive prostatic massage and drug therapy as an alternative to transurethral resection of the prostate. MedGenMed 8: 19, 2006.

17. Horńak M, Bàrdos A and Stefancik J: Effect of drug therapy on lower urinary tract symptoms indicating the presence of benign prostatic hyperplasia in transurethral resection of the prostate. Rozhl Chir 81: 421-424, 2002 (In Slovak).

18. Suzuki M, Ohtake A, Yoshino T, Yuyama H, Hayashi A, Ukai M, Okutsu H, Noguchi Y, Sato S and Sasamata M: Effects of solifenacin succinate (YM905) on detrusor overactivity in conscious cerebral infarcted rats. Eur J Pharmacol 512: 61-66, 2005.
19. Ohtake A, Sato S, Ikeda K, Sasamata M and Miyata K: Pharmacological and clinical profile of solifenacin succinate (Vesicare) developed as a new therapeutic agent for overactive bladder. Nihon Yakurigaku Zasshi 128: 425-432, 2006 (In Japanese)

20. Choo MS, Lee JZ, Lee JB, Kim YH, Jung HC, Lee KS, Kim JC, Seo JT, Paick JS, Kim HJ, et al: Efficacy and safety of solifenacin succinate in Korean patients with overactive bladder: A randomised, prospective, double-blind, multicentre study. Int J Clin Pract 62: 1675-1683, 2008.

21. Macey MR and Raynor MC: Medical and surgical treatment modalities for lower urinary tract symptoms in the male patient secondary to benign prostatic hyperplasia: A review. Semin Intervent Radiol 33: 217-223, 2016.

22. Bishr M, Boehm K, Trudeau V, Tian Z, Dell'Oglio P, Schiffmann J, Jeldres C, Sun M, Shariat SF, Graefen M, et al: Medical management of benign prostatic hyperplasia: Results from a population-based study. Can Urol Assoc J 10: 55-59, 2016.

23. Ozer K, Horsanali MO, Gorgel SN, Horsanali BO and Ozbek E: Association between benign prostatic hyperplasia and neutrophil-lymphocyte ratio, an indicator of inflammation and metabolic syndrome. Urol Int 98: 466-471, 2017.

24. Hu J, Zhang L, Zou L, Hu M, Fan J, Cai Y, Xu G, Fang J, Ding Q and Jiang $\mathrm{H}$ : Role of inflammation in benign prostatic hyperplasia development among Han Chinese: A population-based and single-institutional analysis. Int J Urol 22: 1138-1142, 2015. 